\title{
Effect of Fusarium culmorum infection on survivability of a T-DNA tagged mutant of Arabidopsis thaliana harboring a mutation in the peptide transporter gene At5g46050
}

\author{
TOMasz Warzecha ${ }^{1}$, Dan LundH ${ }^{2}$, Abul Mandal ${ }^{2 *}$ \\ ${ }^{1}$ Department of Plant Breeding and Seed Science, University of Agriculture, Kraków, Poland \\ ${ }^{2}$ School of Life Sciences, University of Skövde, Skövde, Sweden \\ * Corresponding author: abul.mandal@his.se
}

\begin{abstract}
Previously, we have reported a T-DNA tagged mutant (TAG_009) of Arabidopsis thaliana exhibiting a significant sensitivity to biotic stresses. We have also cloned and analyzed the tagged gene At5g46050. Based on bioinformatic and molecular characterization, we proposed that At5g46050 is involved in the transport of peptides participating in plant defense against biotic stresses. To provide further evidence for supporting our proposal, this time we exposed this mutant to Fusarium culmorum, a potential fungal pathogen. Besides TAG_009 line, in our investigations we included two SALK insertion mutants (SALK_003119 and SALK_145209), two wild-type ecotypes (WT_C24 and WT_Col-0) and an additional T-DNA tagged mutant (TAG_197-6) of $A$. thaliana. We have found that the highest degree of leaf damage was exhibited by TAG_009 line (damage score 4.37), whereas the lowest was observed in WT_Col-0 ecotype (damage score 3.43). The highest rate of mortality after eight weeks of inoculation with $F$. culmorum was also observed in TAG_009 line (85.24\%), while the lowest was in WT_Col-0 line (37.22\%). We have also found that plants of SALK_145209 line, despite being infected with Fusarium, produced the highest number of leaves (average 14.17 leaves per plant), whereas the lowest number of leaves was produced by plants of TAG_197-6 line ( average 9.5 leaves per plant). Statistical analyses showed that the differences between the T-DNA tagged line TAG_009 and WT_Col-0 were significant, whereas in comparison with wild-type control plants WT_C24, they were insignificant. Based on these results, we can conclude that the gene we have tagged by using T-DNA-mediated in vivo gene fusion is indeed involved in the plant defense against Fusarium infection.
\end{abstract}

Key words: Fusarium culmorum, Arabidopsis thaliana, T-DNA tagging, disease resistance

\section{Introduction}

Sustainable crop production is a very significant factor in the context of declining natural resources and growing population, particularly in the developing countries. Recent reports presented by the United Nations (UN), the Food and Agricultural Organization (FAO), and the Third World Academy of Sciences (TWAS) indicate that within the next three decades, the world's population will have grown in such a manner that to avoid food shortage its production will have to be increased by at least $60 \%$ (BBC News, 2004; Myers and Kent, 2001). Unfortunately, besides the natural calamities like drought, soil salinity, low temperature, flood etc, the biotic stress factors have severe impacts on global food production. It is estimated that every year approximately one-third of the global plant harvest is lost due to many plant pathogenic diseases and insect injuries. Besides viral and bacterial pathogens, fungal plant pathogens are the major players that contribute to the severe loss of harvest. Despite these biotic or abiotic stress factors, the world will have to solve this problem by creating an improved and sustainable crop production system using new knowledge and techniques that have been developed recently in the area of plant molecular biology.

Plants exhibiting hardiness to severe biotic and abiotic stresses harbor several adaptive and defense mechanisms that are developed from complex metabolic, hormonal and signaling networks (e.g., jasmonic acid JA, ethylene ET, abscisic acid ABA, reactive oxygen species ROS etc.) responsive to these stress conditions. Apart from these networking safeguards, there are other key players involved in plants defense signaling pathways. They are highly conserved mitogen-activated protein kinase (MAPK) cascades and groups of responsive trans- 
cription factors that regulate diverse molecular and biochemical processes in plant cells, such as growth inhibition and stress responses (Kunkel and Brooks, 2002; Mittler, 2002; Blokhina et al., 2003; Dolferus et al., 2003; Wang et al., 2003a; Nürnberger et al., 2004; Shinozaki and Yamaguchi-Shinozaki et al., 2007). The morphology and distribution of visible abiotic and biotic stress symptoms provide a primary diagnostic indication helping to verify the stress types and the plant responses. In addition, it is possible to differentiate the stress symptoms from natural senescence symptoms (Vollenweider and Günthardt-Georg, 2005).

Previously, in order to identify and isolate the genes that might be involved in plants tolerance to biotic stresses, we employed T-DNA-mediated gene tagging in the model plant Arabidopsis thaliana (Karim et al., 2005 and 2006). A. thaliana has become one of the most widely used plants in biological research during the past decades. Its relatively small genome ( $\sim 125 \mathrm{Mbp})$ with a low number of repetitive sequences and a short life cycle are some of the reasons as to why this plant has been adopted as a model system for molecular and genetic studies (Meyerowitz, 1987; Meinke et al., 1998). In addition, the value of Arabidopsis as a model plant increased significantly at the end of the year 2000 when it became the first plant to have its genome sequenced (The Arabidopsis Genome Initiative 2000). T-DNA tagging, on the other hand, has become a powerful approach for identifying new genes and determining gene functions. By inserting a DNA fragment with a known sequence into the plant genome, a knockout mutation in a particular gene can be generated (Alonso et al., 2003; Mandal et al., 1995; Topping et al., 1995; Feldmann, 1991). Knockout of the plant gene may consequently result in plants with a recognizable mutant phenotype. Insertion of the TDNA does not need to occur in the exon of a functional gene to result in a mutant phenotype. Several researchers have shown that insertions can also occur in the introns and in $5^{\prime}$ or 3 ' non-coding regions of the gene leading to a mutant phenotype of the plant (AzpirozLeehan and Feldmann, 1997). Furthermore, the insertional mutagen not only creates a mutation, but also "tags" the affected gene. This enables researchers to identify, isolate and study the gene in question.

This paper is a continuation of our previous research towards cloning and characterization of genes involved in the hardiness of plants to biotic stresses carried out by using T-DNA-mediated gene tagging and in vivo gene fusion in Arabidopsis thaliana (Karim et al., 2005 and 2006). We have isolated a T-DNA tagged mutant (line TAG_009) that exhibits an increased sensitivity to mechanical injuries and infection with bacterial pathogens Erwinia carotovora and Pseudomonas syringae. We have also cloned the T-DNA tagged gene, At5g46050 (AtPTR3), from $A$. thaliana. Based on the results obtained while characterizing the function of this gene, we have postulated that At5g46050 encodes a protein which is involved in transporting peptides required for the developing of the plant hardiness to biotic stresses (Karim et al., 2006). Here we report further evidence supporting our previous postulate regarding the function of the T-DNA tagged gene At5g46050. In order to do so, we exposed the plants of the tagged line TAG_009 to another severe fungal plant pathogen Fusarium culmorum. Besides TAG_009, two other insertion mutants (SALK 003119 and SALK_145209), two wild-type ecotypes (WT_C24 and WT_Col-0) and another T-DNA tagged mutant of $A$. thaliana TAG_197-6 were included in these investigations and used as the control material.

\section{Materials and methods \\ Plant material}

All plant materials used in our study consisted of Arabidopsis thaliana. Mutant lines TAG_009 and TAG 197-6 were generated by T-DNA mediated gene tagging and in vivo gene fusion in ecotype $\mathrm{C} 24$ as described previously (Mandal et al., 1995). Cloning and characterization of the genes tagged in the TAG_009 line (Karim et al., 2005 and 2007) and TAG_197-6 line (Svensson et al., 2004 and 2005) have also been reported. Two insertion mutants, SALK_003119 and SALK_145209 (Alonso et al., 2003) were kindly supplied by the Arabidopsis Stock Center at the SALK Institute, USA (http://www. signal.salk.edu). SALK_ 003119 harbors an insertion mutation almost identical to that in the T-DNA tagged line TAG_009 (gene At5g46050), whereas SALK_145 209 contains a mutation identical to that in the T-DNA tagged line TAG_197-6 (gene At4g20010). Wild-type WT_ Col-0 and WT_C24 ecotypes were obtained from the Swedish University of Agricultural Sciences, Uppsala.

\section{Preparation of plants}

Arabidopsis thaliana seeds were first surface sterilized for 5 minutes with $70 \%$ ethanol and then rinsed 
three times with sterilized water. Under axenic conditions, seeds were then plated on Petri dishes containing $1 \%$ sterile agar medium $(0.2 \mathrm{~g}$ of agar diluted in $20 \mathrm{ml}$ of sterile water). Each plate contained approximately 20 seeds. In order to enhance the germination rate (Hensel et al., 1993; Scholl et al., 1997), the plates were kept in a growth chamber for 7 days at $7^{\circ} \mathrm{C}$ by maintaining 12 -h day (light intensity $130 \mu \mathrm{mol} / \mathrm{m}^{2} / \mathrm{s}$ ).

\section{Preparation of Fusarium culmorum}

Fusarium culmorum(W.G.Sm.) Sacc. strain IPO34801 was kindly supplied by the Plant Protection Institute, Wageningen, The Netherlands. The virulence of this strain has been previously studied in wheat (Wojciechowski et al., 1997) and barley (Chelkowski et al., 2000). Incubation of the fungus was carried out on Petri dishes containing $19 \mathrm{~g}$ of PDA (otato Dextrose Agar, Sigma Chemical Company) per liter. Petri plates were kept in the microbiological growth chamber (Heraeus B 6060) maintaining complete darkness for 7 days at $22^{\circ} \mathrm{C}$.

\section{Leaf damage}

Germinating seedlings (2-4 leaf seedlings) were transferred onto disks ( $\varnothing 2 \mathrm{~mm}$ ) of PDA culture that contained rapidly growing Fusarium culmorum, strain IPO 348-01, and incubated for 7 days at temperature $22^{\circ} \mathrm{C} / 20^{\circ} \mathrm{C}$ (day/ night) using a $12 \mathrm{~h}$ day (light intensity $130 \mu \mathrm{mol} / \mathrm{m}^{2} / \mathrm{s}$ ) with a relative humidity of $100 \%$. Control seedlings were treated with sterile water and allowed to grow under identical growth conditions. The experiment was carried out in a completely randomized design with three replications. Each replication consisted of 40 plants/investigated line. The level of seedling damage caused by Fusarium culmorum infection was evaluated on the basis of a five degree scale (Chełkowski and Manka, 1983) as follows: 1 , very week infection; 2 , weak infection; 3 , medium infection; 4 , high infection and 5 , very high infection.

\section{Mortality of plants}

Germination of $A$. thaliana seeds and cultivation of IPO 348-01 strain of $F$. culmorum were performed in the same way as described above (experiment with leaf infection). After seven days of germination, the seedlings were transferred onto soil pots. The soil was first sterilized by autoclaving. Young roots were then inoculated with disks ( $\varnothing 2 \mathrm{~mm}$ ) of PDA culture of $F$. culmorum (W.G.Sm.) Sacc. isolate IPO348-01. To enhance infection, high humidity was maintained by covering all pots with plastic foils. Plants were kept in the growth chamber under following conditions: temperature 15 to $17^{\circ} \mathrm{C}$, photoperiod $16 \mathrm{~h} /$ day $\left(130 \mu \mathrm{mol} / \mathrm{m}^{2} / \mathrm{s}\right)$, relative humidity $100 \%$. Pots were watered regularly every third day. Mortality of each line was estimated every seventh post-inoculation day based on the difference between the number of inoculated plants and the number of plants that could survive and grow further after eight weeks of infection. The number of leaves per plant was also determined after eight weeks of inoculation. The flowering time was defined as the time lapse between the sowing of the seeds and the appearance of the first flower buds.

\section{Experimental design}

For the estimation of the survival rates of the inoculated plants, we made seven experimental combinations. The experiments were carried out in a completely randomized design with three replications. The data obtained from these observations were calculated statistically. By using the software STATISTICA 7.0 - StatSoft (STATISTICA System Reference, StatSoft 2002), we have performed one factor analysis of variance, ANOVA (Snedecor and Cochran, 1989) to estimate significance of the differences observed between the investigated lines. In some cases, conclusions were made on the basis of least significant differences (LSD) between these lines.

\section{Results}

\section{Leaf damage}

As mentioned in Materials and methods section, the level of leaf damage caused by Fusarium culmorum was estimated seven days after inoculation based on a 5-degree evaluation scale. A variance analysis was performed to estimate the significance of the differences seen in the investigated lines. The results are shown in Table 1. As can be seen the examined lines differ statistically at a significance level $\alpha=0.05$. The data collected in Table 2 indicate that among the mutant plants, the highest degree of leaf damage (score 4.37) was observed in the plants of TAG_009 line, whereas the lowest (score 3.43) was seen in WT_Col-0 line. The level of damage in wildtype plants of ecotype WT_Col-0 differs significantly from that observed in the remaining investigated lines (except for plants of SALK_145209 line, score 3.80). 
The difference in the degree of damage between the wildtype ecotypes WT_C24 (score 3.93) and WT_Col-0 (score 3.43) was also significant as the Least Significant Difference (LSD) at $\alpha=0.01$ for this analysis was 0.48 (Table 2). However, the difference between SALK_145209 and SALK_003119 resulted in LSD 0.40 (less than the wild-type) and remained statistically insignificant (Table 2).

Table 1. Analysis of variance for leaf damage score of Arabidopsis thaliana. Data obtained were calculated statistically by using StatSoft and variance analysis was done by using ANOVA

\begin{tabular}{l|c|c|c|c}
\hline $\begin{array}{c}\text { Source } \\
\text { of variation }\end{array}$ & $\begin{array}{c}\text { Degrees } \\
\text { of freedom }\end{array}$ & $\begin{array}{c}\text { Square } \\
\text { sum }\end{array}$ & $\begin{array}{c}\text { Mean } \\
\text { squares }\end{array}$ & F emp \\
\hline Lines & 5 & 1.6369 & 0.3274 & $4.564^{*}$ \\
\hline Error & 12 & 0.8607 & 0.0717 & \\
\hline Total & 17 & 2.4977 & - & \\
\hline
\end{tabular}

* indicates significant at $\alpha=0.05$

Table 2. Effects of Fusarium culmorum infection on leaf damage in wild type (WT) Arabidopsis thaliana and its mutants (TAG/SALK). The level of damage was estimated after 21 days after inoculation, based on a five-degree scale (1-5). One represents a very low level, whereas five represents a very high level of leaf damage

\begin{tabular}{l|c}
\hline \multicolumn{1}{c|}{ Lines } & Disease score \\
\hline TAG-009 & 4.37 \\
\hline SALK_003119 & 4.20 \\
\hline TAG-197-6 & 4.10 \\
\hline WT_C24 & 3.93 \\
\hline SALK_145209 & 3.80 \\
\hline WT_Col-0 & 3.43 \\
\hline Mean & 3.97 \\
\hline LSD & 0.48 \\
\hline
\end{tabular}

\section{Survival of plants}

The rate of mortality of the transgenic and the WT control plants was estimated on the basis of differences between the number of inoculated plants and the number of plants that could survive infection and grow further as a normal flowering plant. The results obtained from the variance analysis (Table 3 ) revealed that the difference in surviving capacity between plants was highly significant (at $\alpha=0.01$ ). The result presented in Table 4 concerns mortality of plants after eight week of $F u$ sariuminfection. However, we measured mortality every seventh post-inoculation day. We observed that the difference in surviving capacity of WT_Col-0 (37.22\% mortality) and the T-DNA-tagged mutant TAG_009 (85.24\% mortality) was statistically significant as well as between WT_C24 and its mutant TAG_009 (LSD 38.57\%). Plants that survived Fusarium inoculation for a period of eight weeks could grow further and complete all the developmental cycles (Meinke et al., 1998, www.arabidopsis. org).

Table 3. Analysis of variance for mortality of $A$. thaliana lines. Data obtained were calculated statistically by using StatSoft and variance analysis was done by using ANOVA

\begin{tabular}{l|c|c|c|c}
\hline $\begin{array}{c}\text { Source } \\
\text { of variation }\end{array}$ & $\begin{array}{c}\text { Degrees } \\
\text { of freedom }\end{array}$ & $\begin{array}{c}\text { Square } \\
\text { sum }\end{array}$ & $\begin{array}{c}\text { Mean } \\
\text { squares }\end{array}$ & F emp \\
\hline Lines & 5 & 6025.71 & 1205.14 & $7.4503^{* *}$ \\
\hline Error & 12 & 1941.08 & 161.76 & \\
\hline Total & 17 & 7966.80 & - & \\
** indicates significant at $\alpha=0.01$
\end{tabular}

Table 4. Mortality of wild type $A$. thaliana (WT) and its mutants (TAG/SALK) after exposition to Fusarium culmorum. Mortality was estimated in percentages after eight weeks of inoculation on the basis of differences between the number of inoculated plants and the number of plants that could survive this infection and grow further to a normal flowering plant

\begin{tabular}{l|c}
\hline \multicolumn{1}{c|}{ Lines } & Mortality (\%) \\
\hline TAG_009 & 85.24 \\
\hline SALK_145209 & 83.33 \\
\hline SALK_003119 & 66.67 \\
\hline TAG_197-6 & 50.00 \\
\hline WT_C24 & 46.67 \\
\hline WT_Col-0 & 37.22 \\
\hline Mean & 61.52 \\
\hline LSD & 22.63 \\
\hline
\end{tabular}

Table 4 indicates that the lowest rate of mortality was found in plants of WT_Col-0 (37.22\%), whereas the highest in plants of lines TAG_009 and SALK_145209, $85.24 \%$ and $83.33 \%$ mortality, respectively.

However, the difference in the mortality rate between TAG_009 and the insertion mutants SALK_003119 and SALK_145209 remained insignificant. Difference in the lethality in these mutants varied from 85.24 to $66.67 \%$ (Table 4) and was less than the calculated LSD (22.63). 


\section{Development of leaves and flowers}

Plants of all of the investigated ecotypes could develop leaf rosettes after two weeks of germination regardless of the inoculation with $F$. culmorum. However, eight weeks after inoculation, the average number of leaves per plant varied significantly among the genotypes. One-factor analysis of variance, ANOVA (STATISTICA System Reference, StatSoft 2002) conducted for leaf formation revealed that the difference in the number of leaves developed after eight weeks Fusarium infection was highly significant (at $\alpha=0.01$ ) - Table 5. Table 6 indicates that after eight weeks Fusarium infection, the highest number of leaves per plant (14.17) was found for SALK_145209 insertion line, whereas the lowest for TAG_197-6 line (9.5 leaves per plant). Number of leaves/plant in wild type control plants, WT_Col-0 and WT_C24, was 9.67 and 12.7, respectively (Table 6).

Table 5. Analysis of leaf number variance in Arabidopsis thaliana. Data obtained were calculated statistically by using StatSoft and variance analysis was done by using ANOVA

\begin{tabular}{l|c|c|c|c}
\hline $\begin{array}{c}\text { Source } \\
\text { of variation }\end{array}$ & $\begin{array}{c}\text { Degrees } \\
\text { of freedom }\end{array}$ & $\begin{array}{c}\text { Square } \\
\text { sum }\end{array}$ & $\begin{array}{c}\text { Mean } \\
\text { squares }\end{array}$ & F emp \\
\hline Lines & 5 & 52.923 & 10.585 & $6.411^{* *}$ \\
\hline Error & 12 & 19.816 & 1.651 & \\
\hline Total & 17 & 72.74 & - & \\
\hline
\end{tabular}

** indicates significant at $\alpha=0.01$

Table 6. Effects of Fusarium culmorum infection on the leaf production in wild type Arabidopsis thaliana (WT) and its mutants (TAG/SALK). Average number of leaves per plant was estimated eight weeks after inoculation

\begin{tabular}{l|c}
\hline \multicolumn{1}{c|}{ Lines } & Number of leaves \\
\hline SALK_145209 & 14.17 \\
\hline SALK_003119 & 13.08 \\
\hline TAG_009 & 12.46 \\
\hline WT_C24 & 12.17 \\
\hline WT_Col-0 & 9.67 \\
\hline TAG_197-6 & 9.50 \\
\hline Mean & 11.84 \\
\hline LSD & 2.29 \\
\hline
\end{tabular}

The difference in the number of leaves developed during eight weeks Fusarium infection was significant for WT_Col-0 and both SALK mutants as well as for WT_C24 and mutant TAG_197-6 (Table 6). However, the difference in leaf production between TAG-009 and WT_C24 was only 0.29 (which is lower than the calculated LSD 2.29) and therefore, remained insignificant (Table 6). Besides wild type controls (WT_Col-0 and WT_C24) only one mutant line, TAG_197-6, could grow further and produce flowers and fertile seeds (data not shown). Plants of WT_C24 flowered earlier, approximately after 30 days from sowing, whereas the plants of line TAG_197-6 flowered late approximately after 55 days from sowing.

\section{Discussion}

This paper is a part of our T-DNA-mediated gene tagging project towards isolation and characterization of Arabidopsis thaliana genes that might be involved in the tolerance of plants to biotic stresses. Previously, we have reported regarding the identification of the T-DNA tagged mutant TAG_009 and isolation of the tagged gene AtPTR3 (Karim et al., 2005 and 2007). Based on our investigations with a bacterial pathogen Erwinina carotovora we have postulated that the gene we have isolated is involved in the tolerance of plants to bacterial infection and/or mechanical wounding. In this paper, by exposing the mutant line TAG_009 to another potential fungal pathogen Fusarium culmorum, we provide further evidence that supports our previous postulates. In our recent investigations, we have introduced both the T-DNA tagged and the SALK insertion mutants. It should be mentioned here that SALK_003119 and SALK_145209 mutants were generated from WT_Col-0 background (Alonso et al., 2003), whereas TAG_009 and TAG_197-6 mutants were generated from WT_C24 background (Mandal et al., 1995). Therefore, the data obtained in the tagged mutant TAG_009 have been compared with those obtained in wild type WT_C24 controls, whereas the data resulting from the SALK mutants have been compared with those seen in wild type WT_Col-0 controls. The results presented in Table 2 clearly show that when exposed to Fusarium infection, the level of leaf damage in plants of line TAG_009 was insignificantly higher (score 4.37) than that observed in the plants of wild type control WT_C24 (scores 3.93). However, the difference in leaf damage between SALK_003119 (score 4.20) and the wild type WT_Col-0 
(score 3.43) remained significant. Surprisingly, the difference in leaf damage between the WT_C24 (score 3.93) and WT_Col-0 (score 3.43) was also significant. This can be explained by the fact that, as described above, these are two different ecotypes. WT_C24 contains comparatively less trichomes on the surface of the leaves in comparison with the leaves of WT_Col-0. It has been postulated that trichomes are involved in the protection of plants against pathogen infection (Meyerowitz, 1987) and this is why WT_Col-0 ecotype is less susceptible to pathogen infection than WT_C24 ecotype. In addition, lack of trichomes may also be a reason that explains the insignificant difference in leaf damage between WT_C24 and TAG_009.

In our experiments, we have determined the resistance or susceptibility of the investigated lines to $\mathrm{Fu}$ sarium infection based on the differences between the number of inoculated plants and the number of plants that survived this infection, grew further and bloom. The results presented in Table 4 demonstrate that the difference in mortality caused by Fusarium infection between the plants of line TAG_009 (85.24\%) and the wild type WT_C24 controls (46.67\%) was significant. Similar significant difference in mortality rate was also obtained between the plants of SALK_003119 (66.67\% mortality) and WT_Col-0 controls (37.22\% mortality). However, the difference in the mortality rate between the wild type control plants, WT_Col-0 and WT_C24 was insignificant (Tables 3 and 4). Similar experiments concerning susceptibility of $A$. thaliana to several plant pathogenic bacteria or fungi have been previously reported by many researchers. Studies conducted by several researchers revealed that the survival rate of the esa1 mutant when exposed to necrotrophic pathogens (either Alternaria brassicicola, Botrytis cinerea or Plectosphaerella cucumerina) was much lower than that observed in the wild type control plants (Tierens et al., 2002; Kishimoto et al., 2006). Van Hemelrijck et al. (2006) reported that the esa1 mutant was also susceptible to cereal pathogens such as Fusarium culmorum and Fusarium graminearum. These researchers observed that only after nine days of inoculation, the mutant esa1 was much more susceptible to Fusarium infection in comparison with the wild type WT_Col-0 plants. Some reports have also proved that wild type $A$. thaliana, ecotype Columbia (WT_Col-0) is less susceptible to the cereal pathogens $F$. graminearum and $F$. culmorum as com- pared to the esa1 mutant (Urban et al., 2002; Van Hemelrijck et al., 2006). In our studies, the mortality rates observed in the wild type controls was significantly lower than those observed in the respective mutants lines (Table 4).

Our investigations concern not only the mortality rates of the investigated lines when exposed to Fusarium infection but also their capacity to recover from the fungal infection and to grow further to produce flowers and seeds. By comparing the results obtained for SALK_003119 and WT_Col-0 lines, we found that the difference in number of leaves between these two lines was statistically significant. In these lines, the number of leaves per plant was 13.08 and 9.67 , respectively (Table 6). But when comparing the results obtained for TAG_009 and WT_C24, we could not find any statistically significant difference (Table 6). These data do not correlate with our expectation that "the higher the mortality the lower the number of leaves per plant". Our explanation to these results is that the mortality of $A$. thaliana ecotype C24 caused by Fusarium infection and its capacity to develop rosette leaves after recovery from this infection are two separate features and not necessarily correlated. We should remember that both lines WT_C24 and TAG_009 are derived from C24 background and contain less trichomes on the leaf surface than the Col-0 ecotype. Trichomes may also play a significant role in developing rosette leaves in C24 ecotype. Moreover, we observed that the plants of the mutant line TAG_197-6 exhibited a late flowering phenotype in comparison with the WT_C24 controls. The flowering time occurred approximately after 55 and 30 days, respectively. These results are almost identical to those we have reported previously (Svensson et al., 2004 and 2005).

In conclusion, the T-DNA tagged mutant TAG_009 that we isolated and characterized previously also exhibits a higher degree of susceptibility towards Fusarium culmorum infection. The degree of TAG 009 susceptibility to this fungus remains in agreement with the study we conducted with bacterial infection, particularly with E. carotovora (Karim et al., 2005 and 2006). This confirms our previous postulate that the mutant we have identified is less resistant to pathogenic infection (either bacterial or fungal) in comparison with the wild type control plants. Secondly, it supports our previous postulate concerning the involvement of the tagged gene in plants defence against biotic stresses. 


\section{Acknowledgements}

This research was supported jointly by FORMAS (The Swedish Research Council for Environment, Agricultural Sciences and Spatial Planning) and SIDA (The Swedish International Development Cooperation Agency). We are also grateful for the grant from the Nilsson-Ehle Foundation.

\section{References}

Alonso J.M., Stepanova A.N., Leisse T.J., Kim C.J., Chen H., Shinn P., Stevenson D.K., Zimmerman J., Barajas P., Cheuk R., Gadrinab C., Heller C., Jeske A., Koesema E., Meyers C.C., Parker H., Prednis L., Ansari Y., Choy N., Deen H., Geralt M., Hazari N., Hom E., Karnes M., Mulholland C., Ndubaku R., Schmidt I., Guzman P., AguilarHenonin L., Schmid M., Weigel D., Carter D.E., Marchand T., Risseeuw E., Brogden D., Zek A., Crosby W.L., Berry C.C., Ecker J.R. (2003) Genome-wide insertional mutagenesis of Arabidopsis thaliana. Science 301: 653657.

Azpiroz-Leehan R., Feldmann K.A. (1997) T-DNA insertion mutagenesis in Arabidopsis: going back and forth. Trends Gen. 13: 152-156.

BBC News. 2004. Viewpoints: Food for all? Published: 2004/ 11/27, 13:54:33 GMT. http://news.bbc.co.uk/go/pr/fr/-/hi/ science/nature/4038189.stm. BBC, MMVII, London, UK

Blokhina O., Virolainen E., Fagerstedt K.V. (2003). Antioxidants, oxidative damage and oxygen deprivation stress: a review. Ann. Bot. 91: 179-194.

Chełkowski J., Mańka M. (1983) The ability of Fusaria pathogenic to wheat, barley and corn produce zearalenone. Phytopath. Z. 106: 354-359.

Chełkowski J., Wiśniewska H., Adamski T., Goliński P., Kaczmarek Z., Kostecki M., Perkowski J., Surma M. (2000) Effect of Fusarium culmorum head blight on mycotoxin accumulation and yield traits in barley double haploids. J. Phytophatology 148: 541-545.

Dolferus R., Klok E.J., Delessert C., Wilson S., Ismond K.P., Good A.G., Peacock W.J., Dennis E.S. (2003) Enhancing the anaerobic response. Ann. Bot. 91: 111-117.

Feldmann K.A. (1991) T-DNA insertion mutagenesis in Arabidopsis: mutational spectrum. Plant J. 1: 71-82.

Hensel L.L., Grbic V., Baumgarten D.A., Bleecker A.B. (1993) Development and age-related processes that influence the longevity and senescence of photosynthetic tissues in Arabidopsis. Plant Cell 5: 553-564.

Karim S., Holmström K.O., Mandal A., Dahl P., Homann S., Brader G., Palva E.T., Pirhonen M. (2007) AtPTR3, awound-induced peptide transporter needed for defence against virulent bacterial pathogens in Arabidopsis. Planta (DOI:10:1007/s00425-006-0451-5).

Karim S., Lundh D., Holmström K.O., Mandal A., Pirhonen M. (2005) Structural and functional characterization of AtPTR3, a stress-induced peptide transporter of Arabidopsis. J. Mol. Modeling 11: 226-236.
Kishimoto K., Matsui K., Ozawa R., Takabayashi J. (2006) Components of C6-aldehyde-induced resistance in Arabidopsis thaliana against a necrotrophic fungal pathogen, Botrytis cinerea. Plant Sci. 170: 715-723.

Kunkel B.N., Brooks D.M. (2002) Cross talk between signalling pathways in pathogen defence. Curr. Opin. Plant Biol. 5: 325-331.

Mandal A., Sandgren M., Holmström K.O., Gallois P., Palva E.T. (1995) Identification of Arabidopsis thaliana sequences responsive to low temperature and abscisic acid by T-DNA tagging and in vivo gene fusion. Plant Mol. Biol. Rep. 13: 243-254.

Meinke D.W., Cherry J.M., Dean C., Rounsley S.D., Koornneef M. (1998) Arabidopsis thaliana: A model plant for genome analysis. Science 282(5389): 662-682.

Meyerowitz E.M. (1987) Arabidopsis thaliana. Ann. Rev. Gen. 21: $93-111$.

Mittler R. (2002) Oxidative stress, antioxidants and stress tolerance. Trends Plant Sci. 7: 405-410.

Myers N., Kent J. (2001) Water demand and supply. In: Perverse subsidies: How tax dollars can undercut the environment and the economy. (p. 123). Island Press, London.

Nürnberger T., Brunner F., Kemmerling B., Piater L. (2004) Innate immunity in plants and animals: striking similarities and obvious differences. Immunol. Rev. 198:249-266.

Scholl R., Rivero-Lepinckas L., Crist D. (1997) Growth of Plant and Preservation of Seeds. [In:] Arabidopsis Protocols eds. Martinez-Zapater J.M., Salinas J., Meth. Mol. Biol. 82: 1-12.

Shinozaki K., Yamaguchi-Shinozaki K. (2007) Gene networks involved in drought stress response and tolerance. J. Exp. Bot. 58: 221-227.

Snedecor G.W., Cochran W.G. (1989) Statistical Methods. Iowa State University Press. p. 217-235.

Svensson M., Lundh D., Bergman P., Mandal A. (2005) Characterization of a T-DNA tagged gene of Arabidopsis thaliana that regulates gibberellin metabolism and flowering time. Funct. Plant Biol. 32: 923-932.

Svensson M., Lundh D., Ejdebäck M., Mandal A. (2004) Functional prediction of a T-DNA tagged gene of Arabidopsis thaliana by in silico analysis. J. Mol. Modeling 10: 130-138.

The Arabidopsis Genome Initiative. 2000. Analysis of the genome sequence of the flowering plant Arabidopsis thaliana. Nature 408: 796-815.

Tierens K.F.M.J., Thomma B.P.H.J., Bari R.P., Garmier M., Eggermont K., Brouwer M, Penninckx I.A.M.A., Broekaert W.F., Cammue B.P.A. (2002) Esa1, an Arabidopsis mutant with enhanced susceptibility to a range of necrotrophic fungal pathogens, shows a distorted induction of defense responses by reactive oxygen generated compounds. Plant J. 29: 131-140.

Topping J., Lindsey K. (1995) Insertional mutagenesis and promoter trapping in plants for the isolation of genes and the study of development. Transgenic Res. 4: 291-305.

Urban M., Daniels S., Mott E., Hammond-Kosack K. (2002) Arabidopsis is susceptible to the cereal ear blight fungal 
pathogens Fusarium graminearum and Fusarium culmorum. Plant J. 32: 961-973.

Van Hemelrijck W., Wouters P.F.W., Brouwer M., Windelinckx A., Goderis I.J.W.M., De Bolle M.F.C., Thomma B.P.H.J., Cammue B.P.A., Delauré S.L. (2006) The Arabidopsis defense response mutant esa1 as a model to discover novel resistance traits against Fusarium diseases. Plant Sci. 171: 585-595.

Vollenweider P., Günthardt-Georg M.S. (2005) Diagnosis of abiotic and biotic stress factors using the visible symptoms in foliage. Environ. Pollut. 137: 455-465.
Wang W., Vinocur B., Altman A. (2003) Plant responses to drought. Salinity and extreme temperatures: towards genetic engineering for stress tolerance. Planta 218: 1-14.

Wojciechowski S., Chełkowski J., Ponitka A., Ślusarkiewicz-Jarzina A. (1997) Evaluation of spring and winter wheat reaction to Fusarium culmorum and Fusarium avenaceum. J. Phytophatol. 145: 99-103. 